\title{
Assessment of Insulin Action and Glucose Effectiveness in Diabetic and Nondiabetic Humans
}

\author{
A. A. Alzaid, S. F. Dinneen, D. J. Turk, A. Caumo, ${ }^{\ddagger}$ C. Cobelli, ${ }^{\star}$ and R. A. Rizza \\ Endocrine Research Unit, Department of Medicine, Rochester, Minnesota 55905; *Department of Electronics and Informatics, \\ University of Padua, Italy; and ${ }^{\ddagger}$ San Raffaele Scientific Institute, Milan, Italy
}

\begin{abstract}
Insulin concentrations in humans continuously change and typically increase only when glucose also increases such as with eating. In this setting, it is not known whether the severity of hepatic and extrahepatic insulin resistance is comparable and whether the ability of glucose to regulate its own uptake and release is defective in non-insulin-dependent diabetes mellitus (NIDDM). To address this question, NIDDM and nondiabetic subjects were studied when glucose concentrations were clamped at either $5 \mathrm{mM}$ (euglycemia) or varied so as to mimic the glucose concentrations observed in nondiabetic humans after food ingestion (hyperglycemia). Insulin was infused so as to simulate a "nondiabetic" postprandial profile. During euglycemia, insulin increased glucose disposal in nondiabetic but not diabetic subjects indicating marked extrahepatic resistance. In contrast, insulin-induced suppression of glucose release was only minimally less $(P<0.05)$ in diabetic than nondiabetic subjects $\left(-1.06 \pm 0.09\right.$ vs. $-1.47 \pm 0.21 \mathrm{nmol} \cdot \mathrm{kg}^{-1}$ per $\left.4 \mathrm{~h}\right)$. Hyperglycemia substantially enhanced disposal in both groups. Glucose effectiveness measured as the magnitude of enhancement of disposal $\left(0.59 \pm 0.18\right.$ vs. $0.62 \pm 0.17 \mathrm{nmol} \cdot \mathrm{kg}^{-1}$ per 4 h) and suppression of release $(-0.36 \pm 0.12$ vs. $-0.14 \pm 0.12$ $\mathrm{nmol} \cdot \mathrm{kg}^{-1}$ per $4 \mathrm{~h}$ ) did not differ in the diabetic and nondiabetic subjects. In conclusion, when assessed in the presence of a physiological insulin profile, people with NIDDM demonstrate: (a) profound extrahepatic insulin resistance, $(b)$ modest hepatic insulin resistance, and $(c)$ normal ability of glucose to stimulate its own uptake and suppress its own release. (J. Clin. Invest. 1994. 94:2341-2348.) Key words: insulin action - glucose effectiveness - glucose disposal • hepatic glucose release $\cdot$ diabetes mellitus
\end{abstract}

\section{Introduction}

Non-insulin-dependent diabetes mellitus (NIDDM) ${ }^{1}$ is characterized by defects in insulin secretion and action (1-7). The

This study was presented in part at the Annual Meeting of the American Diabetes Association in Las Vegas, NV on 10-15 June 1993.

Address correspondence to Robert A. Rizza, M. D., Endocrine Research Unit, 5-164 West Joseph, Mayo Clinic, Rochester, MN 55905. Dr. Aus Alzaid's current address is Department of Medicine, Armed Forces Hospital, Riyadh 11159, Saudi Arabia.

Received for publication 1 April 1994 and in revised form 12 August 1994.

1. Abbreviations used in this paper: A-V, arterial-venous; NIDDM, non-insulin-dependent diabetes mellitus.

The Journal of Clinical Investigation, Inc.

Volume 94, December 1994, 2341-2348 relative contribution of these defects to the excessive postprandial hepatic glucose release and the inappropriately low glucose uptake that is characteristic of NIDDM is not known. Numerous studies have suggested that insulin-induced suppression of hepatic glucose release and stimulation of glucose disappearance are impaired in NIDDM (2-7). Most of these studies have obtained this information by using either hyperinsulinemic or hyperglycemic clamps to evaluate steady state glucose metabolism in the presence of constant glucose and insulin concentrations (2-7). However, recent studies have demonstrated that changing insulin concentrations have greater effects on glucose metabolism than constant insulin concentrations (8-10). Furthermore, under the conditions of daily living, insulin rarely if ever increases without a concurrent increase in glucose (1). This increase in plasma glucose may facilitate insulin-induced suppression of hepatic glucose release $(11-13)$ and stimulation of tissue glucose uptake $(15,16)$. However, because the effects of glucose on its own metabolism varies depending on the prevailing insulin concentration, it is difficult to determine from conventional hyperglycemic and/or hyperinsulinemic clamps the extent to which the postprandial rise in glucose can compensate for a decrease in insulin action.

The current studies therefore were undertaken to determine whether in the presence of continuously changing insulin and glucose concentrations the severity of hepatic and extrahepatic insulin resistance were comparable and whether the effectiveness of glucose per se was impaired in patients with NIDDM. To examine this question, insulin action was assessed in diabetic and nondiabetic subjects in the presence of insulin concentrations that mimic those typically observed in healthy nondiabetic subjects after carbohydrate ingestion (17). Glucose concentrations either were maintained constant at euglycemic levels so as to measure the effects of insulin per se or were varied to simulate postprandial glucose concentrations so as to measure the combined effects of glucose and insulin. The difference between rates of glucose disposal and release observed during the euglycemic and hyperglycemic experiments was used to determine the extent to which glucose per se modulated its own metabolism.

\section{Methods}

Subjects. After approval by the Mayo Institutional Review Board, 11 subjects with NIDDM and 10 nondiabetic subjects gave informed written consent to participate in the study. Subjects were matched for the characteristics listed in Table I including age, gender, and obesity. The duration of diabetes was $5.1 \pm 1.1 \mathrm{yr}$ (mean \pm SEM) at the time of study. Seven patients were treated with sulfonylureas and four with diet alone. Sulfonylurea treatment was discontinued $3 \mathrm{wk}$ before study. All subjects were in good health, had normal blood pressure, and were at stable weight. None regularly engaged in vigorous aerobic exercise or were taking any medications other than sulfonylureas. The nondiabetic subjects did not have a family history of diabetes. 
Table I. Subject Characteristics

\begin{tabular}{lcc}
\hline & $\begin{array}{c}\text { Nondiabetic } \\
\text { subjects }\end{array}$ & $\begin{array}{c}\text { NIDDM } \\
\text { subjects }\end{array}$ \\
\hline Number & 10 & 11 \\
Sex (male/female) & $5 / 5$ & $8 / 3$ \\
Age (y) & $55 \pm 2$ & $54 \pm 2.3$ \\
Body mass index $\left(\mathrm{kg} / \mathrm{m}^{2}\right)$ & $28.4 \pm 1$ & $2.9 \pm 1.0$ \\
Waist-hip ratio & $0.90 \pm 0.02$ & $0.94 \pm 0.02$ \\
Body fat $(\%)$ & $34 \pm 3.0$ & $32 \pm 3$ \\
Fasting plasma glucose $(\mathrm{mM})$ & $5.3 \pm 0.05$ & $12.4 \pm 1.2^{*}$ \\
Glycosylated $\mathrm{HbA}_{1}(\%)$ & - & $10.3 \pm 0.8$ \\
\hline
\end{tabular}

$* P<0.0001$ vs. control subjects.

Experimental design. Subjects were admitted to the Clinical Research Center at 1700 hour on the evening before study. A standard meal ( $636 \mathrm{kcal}$; $48 \%$ carbohydrate, $32 \%$ fat, $19 \%$ protein) was eaten between 1730 and 1800 hours after which subjects were fasted until the end of the study. A 18-gauge catheter was inserted into a vein of each forearm on the evening of admission. One was connected to a multiport infusion set (Burro Medical Inc., Bethlehem, PA) and used for all study infusions. The other cannula was used overnight for blood sampling. At the time of meal ingestion an infusion of $0.9 \%$ saline (nondiabetic) or insulin ( $1 \mathrm{U} / 10 \mathrm{ml}$ of saline containing albumin) was started. The insulin infusion was adjusted according to the algorithm of White et al. (18) to maintain nocturnal glucose concentrations in the diabetic subjects at concentrations of $\sim 5 \mathrm{mM}$. On the following morning the venous sampling cannula was removed and a cannula was placed retrogradely into a dorsal hand vein, which was inserted into a heated plexiglass box and maintained at $55^{\circ} \mathrm{C}$ to allow intermittent sampling of arterialized venous blood. An additional cannula was placed retrogradely into an antecubital vein of the contralateral forearm to allow intermittent sampling of deep venous blood.

At 0700 hours the next morning, infusions of somatostatin $(2.2 \mu \mathrm{g} /$ $\mathrm{m}^{2}$ per minute), growth hormone $\left(115 \mathrm{ng} / \mathrm{m}^{2}\right.$ per $\mathrm{min}$ ), and glucagon $\left(25 \mathrm{ng} / \mathrm{m}^{2}\right.$ per minute) were started in both groups. An insulin infusion also was started in the nondiabetic subjects. Plasma glucose determinations were performed at 5-min intervals using a glucose oxidase method (Yellow Springs Instrument Co., Yellow Springs, $\mathrm{OH}$ ) and the insulin infusion was adjusted in both groups to maintain glucose at $\sim 5 \mathrm{mM}$, until 1030 hours when the rate was fixed at a constant rate for the remainder of the study. A primed-continuous infusion of $\left[6-{ }^{14} \mathrm{C}\right]$ glucose ( $3 \mu \mathrm{Ci}$ prime, $0.03 \mu \mathrm{Ci} / \mathrm{min}$ continuous) was started at 0900 hours. [6${ }^{3} \mathrm{H}$ ]Glucose also was infused as part of a separate protocol. An additional insulin infusion was started at $1100 \mathrm{~h}$ in all studies. As previously described (19), this so-called prandial insulin infusion was given by a programmable infusion pump (model 22; Harvard Apparatus, South Natick, MA) regulated by an on-line computer (PS2/30 265; International Business Machines, Rochester, MN). The infusion rate was changed every $10 \mathrm{~s}$ so as to deliver insulin in a pattern that mimicked the postprandial insulin concentrations previously observed in healthy nondiabetic subjects (17). The prandial insulin infusion resulted in the delivery of $1.01 \pm 0.01 \mathrm{U} / \mathrm{m}^{2}$ body surface area (or $\sim 2 \mathrm{U}$ per study).

Sufficient glucose was infused to maintain euglycemia on one occasion while on a second occasion a "prandial" glucose profile was mimicked as previously described (19). To maintain glucose specific activity constant, all infused glucose contained $\left[6-{ }^{14} \mathrm{C}\right]$ glucose $(20)$. As previously discussed (7), the rate of the basal $\left[6-{ }^{14} \mathrm{C}\right]$ glucose infusion was adjusted $(-120-0 \mathrm{~min}, 100 \% ; 0-15 \mathrm{~min}, 70 \% ; 15-75 \mathrm{~min}$, $30 \%$; $75-120 \mathrm{~min}, 60 \% ; 120-240 \mathrm{~min}, 80 \%$ of basal) in an attempt to reproduce the anticipated pattern of change of hepatic glucose release to minimize changes in plasma glucose specific activity. The order of study was random. Euglycemic studies were performed in all subjects; nine nondiabetic and eight diabetic subjects also participated in the hyperglycemic studies.

Analytical techniques. Arterialized plasma samples were placed on ice, centrifuged at $4^{\circ} \mathrm{C}$, separated, and stored at $-20^{\circ} \mathrm{C}$ until assay. Plasma insulin, C-peptide, glucagon, growth hormone, and glucose specific activity were measured as previously described $(21,22)$. Body fat composition was determined by dual-energy $\mathrm{x}$-ray absorptiometry (DPX scanner; Lunar Corporation, Madison, WI). Glycosylated hemoglobin concentration was measured by affinity chromatography (Gly-Affin; Isolab, Akron, $\mathrm{OH}$; normal range, 4-7\%).

Calculations. Glucose specific activity was smoothed using the method of Bradley et al. (23). Glucose appearance and disappearance were calculated using Radziuk et al.'s two-compartment model (24). Transfer constants in the basal state were estimated by nonlinear least squares (25) using the data obtained after the priming dose of [6${ }^{14} \mathrm{C}$ ] glucose. Hepatic glucose release was calculated by subtracting the glucose infusion rate from the tracer determined rate of glucose appearance. Arterial-venous ( $\mathrm{A}-\mathrm{V}$ ) difference was calculated by subtracting the glucose concentration present in deep venous blood from that present in simultaneously obtained arterialized blood. Because forearm blood flow did not differ before and after the prandial insulin infusion in the diabetic or nondiabetic subjects on either the euglycemic (diabetic, $3.49 \pm 0.29$ vs. $3.39 \pm 0.27$; nondiabetic, $2.01 \pm 0.32$ vs. $1.97 \pm 0.33 \mathrm{ml} /$ $100 \mathrm{ml}$ forearm) or hyperglycemic (diabetic, $2.71 \pm 0.37$ vs. $2.6 \pm 0.40$; nondiabetic, $2.43 \pm 0.27$ vs. $2.28 \pm 0.39 \mathrm{ml} / 100 \mathrm{ml}$ forearm) study days and because there was considerable intraindividual variability in blood flow during the experiment, data are presented in the text and figures as $\mathrm{A}-\mathrm{V}$ glucose difference rather than forearm glucose uptake. Glucose effectiveness was calculated by subtracting the results observed during euglycemia in each individual from those observed in the same individual during hyperglycemia.

Statistical analysis. Data in text and figures are expressed as the mean \pm SEM. Total integrated responses and responses above basal were calculated using the trapezoidal rule. Total integrated response was defined as total area above zero during the prandial insulin infusion. Area above basal was defined as the area above the mean of the values present during the $30 \mathrm{~min}$ before the prandial insulin infusion. Results between groups (diabetic vs. nondiabetic) and within a group (hyperglycemic vs. euglycemic) were compared using nonpaired and paired Student's $t$ test, respectively. One-tailed tests were used to test the hypotheses that hepatic glucose release was greater and that glucose disposal was lower. All other tests were two tailed. $P<0.05$ was considered statistically significant.

\section{Results}

Glucose and insulin concentrations. Glucose concentrations did not differ in the diabetic and nondiabetic subjects during the basal period (i.e., during the $30 \mathrm{~min}$ before the prandial insulin infusion) of either the euglycemic or hyperglycemic studies (Fig. 1). During the prandial insulin infusions, glucose concentrations in both groups were either maintained constant $(\sim 5$ $\mathrm{mM}$ ) or varied so as to mimic a postprandial rise in glucose $(1,17)$. Glucose concentrations did not differ in the diabetic and nondiabetic subjects during either prandial insulin infusion.

The insulin concentrations present during the basal period were slightly but not significantly higher in the diabetic than nondiabetic subjects during both the euglycemic (126 \pm 18 vs. $87 \pm 8 \mathrm{pmol} /$ liter) and hyperglycemic ( $124 \pm 28$ vs. $88 \pm 12$ $\mathrm{pmol} / \mathrm{liter}$ ) studies. The increment above basal during the prandial insulin infusion was the same in the diabetic and nondiabetic subjects during both the euglycemic ( $8.8 \pm 0.9$ vs. $8.7 \pm 1.3$ $\mathrm{nmol} / \mathrm{liter}$ per $4 \mathrm{~h})$ and hyperglycemic $(9.6 \pm 1.2$ vs. $8.3 \pm 0.9$ nmol/liter per $4 \mathrm{~h}$ ) studies.

C-peptide, glucagon, and growth hormone concentrations. 


\section{Euglycemia Hyperglycomia}
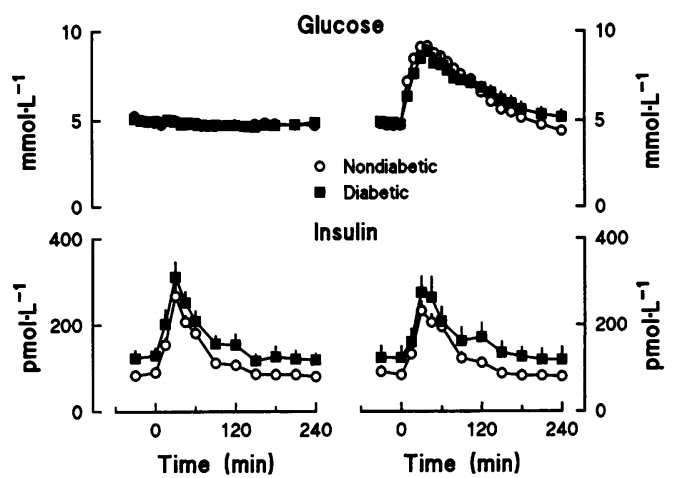

Figure 1. Plasma glucose and insulin concentrations observed during the euglycemic (left) and hyperglycemic (right) studies in the diabetic and nondiabetic subjects. The prandial insulin infusion was started at time 0 .

C-peptide, glucagon, and growth hormone concentrations did not differ in the diabetic and nondiabetic subjects on either the euglycemic or hyperglycemic study days (Fig. 2).

Rates of glucose infusion and glucose specific activity. The glucose infusion rates required to maintain euglycemia $\left(0.87 \pm 0.11\right.$ vs. $1.94 \pm 0.19 \mathrm{mmol} \cdot \mathrm{kg}^{-1}$ per $\left.4 \mathrm{~h}\right)$ or to reproduce the prandial glucose profile $(1.70 \pm 0.26$ vs. $2.70 \pm 0.28$ $\mathrm{mmol} \cdot \mathrm{kg}^{-1}$ per $\left.4 \mathrm{~h}\right)$ were lower $(P<0.02)$ in the diabetic than nondiabetic subjects, respectively (Fig. 3). Plasma glucose specific activity increased sightly but comparably in both groups during both studies.

Glucose disappearance and forearm $A-V$ glucose difference. During the euglycemic study, whereas the prandial insulin infusion caused a substantial increase in both glucose disappearance and $\mathrm{A}-\mathrm{V}$ difference in the nondiabetic subjects, it had no effect on either of these parameters in the diabetic subjects (Fig. 4 , left $)$. Glucose disappearance was lower $(P<0.05)$ in the diabetic than nondiabetic subjects during the prandial insulin infusion whether expressed as the increase above basal $\left(-0.18 \pm 0.09\right.$ vs. $0.49 \pm 0.24 \mathrm{mmol} \cdot \mathrm{kg}^{-1}$ per $\left.4 \mathrm{~h}\right)$ or as the total response $\left(2.19 \pm 0.14\right.$ vs. $3.08 \pm 0.23 \mathrm{mmol} \cdot \mathrm{kg}^{-1}$ per $\left.4 \mathrm{~h}\right)$.

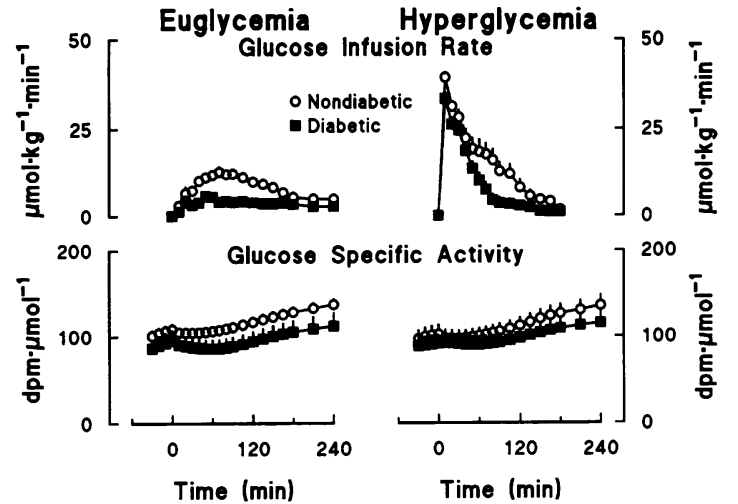

Figure 3. Glucose infusion rates required to achieve target glucose concentrations and glucose specific activities observed in the diabetic and nondiabetic subjects during the euglycemic (left) and hyperglycemic (right) studies. The prandial insulin infusion was started at time 0 .

Similarly the increase in $\mathrm{A}-\mathrm{V}$ glucose difference was lower $(P<0.01)$ in the diabetic than nondiabetic subjects whether expressed as the increase above basal ( $-9 \pm 14$ vs. $78 \pm 15$ $\mathrm{mmol} /$ liter per $4 \mathrm{~h}$ ) or the total response (33 \pm 11 vs. $125 \pm 21$ mmol/liter per $4 \mathrm{~h}$ ).

During the hyperglycemic study, the prandial insulin infusion resulted in a substantial increase in glucose disappearance and $\mathrm{A}-\mathrm{V}$ glucose difference in both groups (Fig. 4, right). However, both glucose disappearance (area above basal, $0.36 \pm 0.26$ vs. $0.97 \pm 0.18 \mathrm{mmol} \cdot \mathrm{kg}^{-1}$ per $4 \mathrm{~h}$; total response, $2.73 \pm 0.21$ vs. $3.69 \pm 0.32 \mathrm{nmol} \cdot \mathrm{kg}^{-1}$ per $4 \mathrm{~h}$ ) and $\mathrm{A}-\mathrm{V}$ glucose difference (area above basal, $33 \pm 11$ vs. $93 \pm 21 \mathrm{mmol} /$ liter per $4 \mathrm{~h}$; total response, $86 \pm 19$ vs. $178 \pm 43 \mathrm{mmol} /$ liter per $4 \mathrm{~h}$ ) remained lower $(P<0.05)$ in the diabetic than nondiabetic subjects.

Hepatic glucose release. Consistent with the higher basal insulin concentrations, basal rates of hepatic glucose release were slightly but not significantly lower in the diabetic than nondiabetic subjects during both the euglycemic $(9.9 \pm 0.5 \mathrm{vs}$. $10.8 \pm 0.9 \mu \mathrm{mol} \cdot \mathrm{kg}^{-1} /$ minute, $\left.P=0.37\right)$ and the hyperglycemic $\left(9.8 \pm 0.7\right.$ vs. $11.3 \pm 0.8 \mu \mathrm{mol} \cdot \mathrm{kg}^{-1}$ per minute, $\left.P=0.16\right)$ stud-

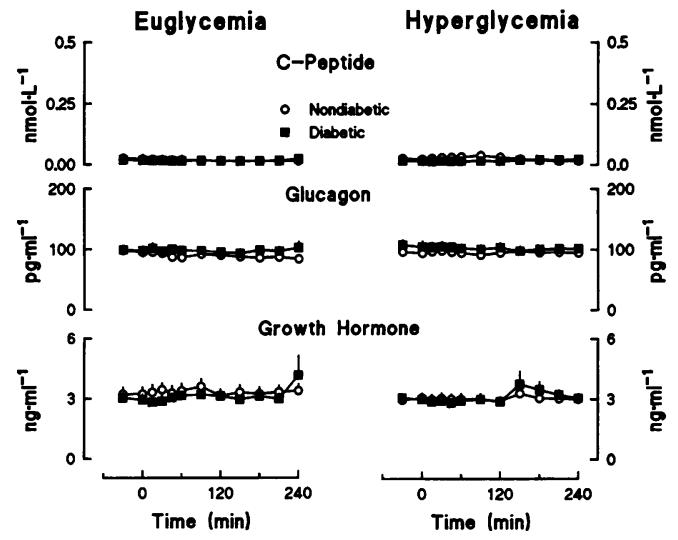

Figure 2. Plasma C-peptide, glucagon, and growth hormone concentrations observed in the diabetic and nondiabetic subjects during the euglycemic (left) and hyperglycemic (right) studies. The prandial insulin infusion was started at time 0 .

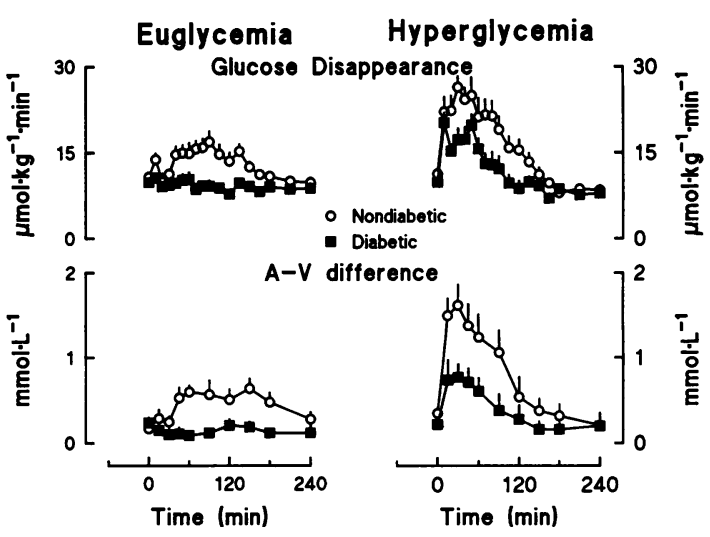

Figure 4. Rates of glucose disappearance and forearm A-V difference observed in the diabetic and nondiabetic subjects during the euglycemic (left) and hyperglycemic (right) studies. The prandial insulin infusion was started at time 0 . 


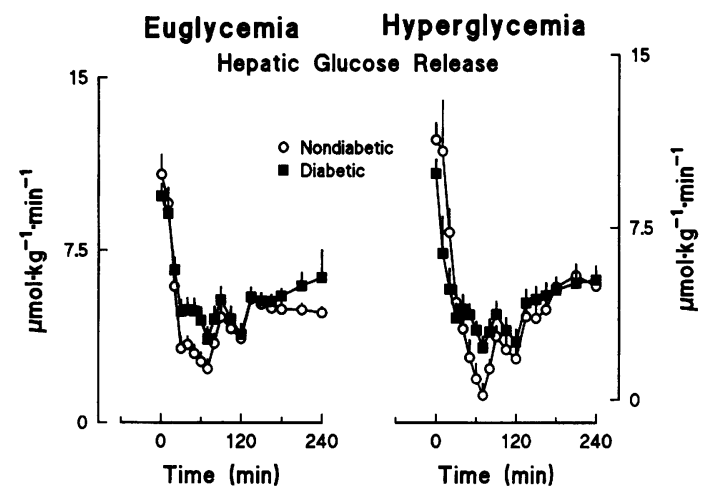

Figure 5. Rates of hepatic glucose release observed in the diabetic and nondiabetic subjects during the euglycemic (left) and hyperglycemic (right) studies. The prandial insulin infusion was started at time 0 .

ies (Fig. 5). Although the prandial insulin infusion resulted in prompt suppression of hepatic glucose release in both groups on both occasions, the degree of suppression was less ( $P$ $<0.05)$ in the diabetic than nondiabetic subjects during the euglycemic $\left(-1.06 \pm 0.09\right.$ vs. $-1.47 \pm 0.21 \mathrm{nmol} \cdot \mathrm{kg}^{-1}$ per $\left.4 \mathrm{~h}\right)$ and the hyperglycemic $(-1.34 \pm 0.16$ vs. $-1.76 \pm 0.14$ $\mathrm{nmol} \cdot \mathrm{kg}^{-1}$ per $4 \mathrm{~h}$ ) experiments. Because the increment in insulin concentrations was the same in both groups, this indicated the presence of insulin resistance.

The effect of glucose per se on glucose disappearance and hepatic glucose release. To examine the effects of glucose per se on glucose disappearance and hepatic glucose release, results in the eight diabetic and nine nondiabetic subjects who underwent both hyperglycemic and euglycemic studies were analyzed. Although glucose concentrations by design differed, as is evident from Fig. 1, insulin concentrations were virtually identical during the hyperglycemic and euglycemic experiments in both the diabetic and nondiabetic subjects. C-peptide, glucagon, and growth hormone concentrations also were the same during both studies in both groups.

Hyperglycemia substantially increased glucose disappearance and forearm $\mathrm{A}-\mathrm{V}$ glucose difference in the diabetic and nondiabetic subjects (see Fig. 6, left and middle). Although hyperglycemia also enhanced suppression of hepatic glucose

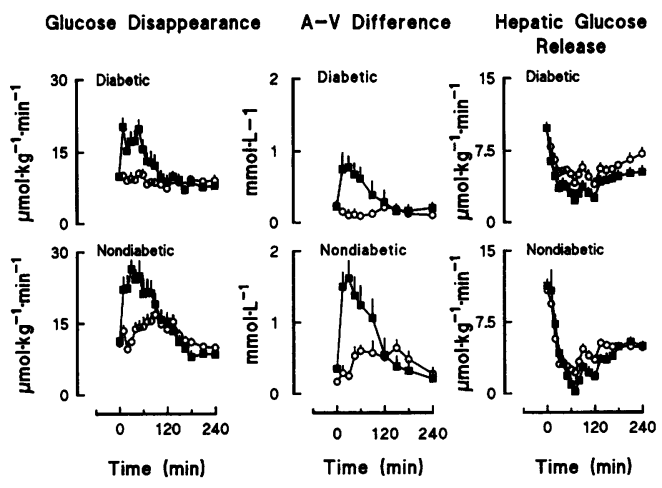

Figure 6. Rates of glucose disappearance, forearm A-V difference, and hepatic glucose release observed in the diabetic (top) and nondiabetic subjects (bottom) during the euglycemic (open circles) and hyperglycemic (solid squares) studies. The prandial insulin infusion was started at time 0 .

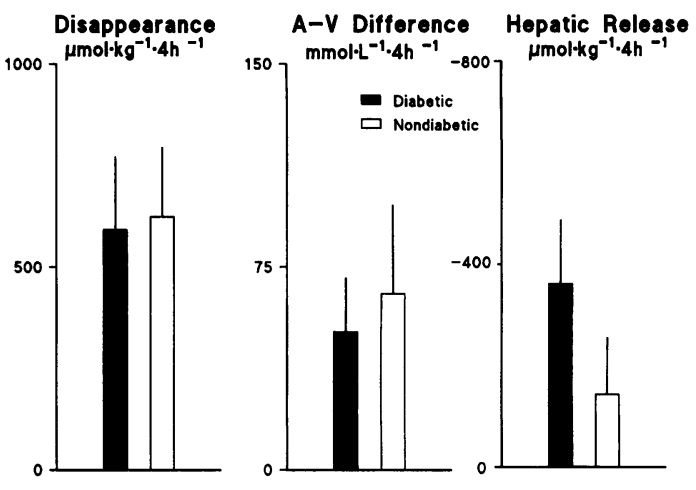

Figure 7. The effects of glucose per se on glucose disappearance (left), forearm $\mathrm{A}-\mathrm{V}$ glucose difference (middle), and hepatic glucose release (right) were determined by subtracting the rates observed in each individual during the euglycemic from those observed in the same individual during the hyperglycemic studies. Results observed in the diabetic subjects are shown as open bars and results observed in the nondiabetic subjects are shown as closed bars.

release, this effect was relatively small in both groups (Fig. 7, right). To determine whether the ability of glucose to facilitate its own uptake and suppress it own release was impaired in NIDDM, the increment above basal during euglycemia in each individual was subtracted from that observed in the same individual during hyperglycemia. As shown in Fig. 7, the ability of glucose to stimulate glucose disposal did not differ in the diabetic and nondiabetic subjects whether measured isotopically, as glucose disappearance $\left(0.59 \pm 0.18\right.$ vs. $0.62 \pm 0.17 \mathrm{nmol} \cdot \mathrm{kg}^{-1}$ per $4 \mathrm{~h}$ ) or as forearm $\mathrm{A}-\mathrm{V}$ glucose difference $(51 \pm 20 \mathrm{vs}$. $65 \pm 33 \mathrm{mmol} /$ liter per $4 \mathrm{~h}$ ). In addition, the ability of glucose to inhibit hepatic glucose release if anything was slightly greater in the diabetic than nondiabetic subjects $(-0.36 \pm 0.13$ vs. $-0.14 \pm 0.11 \mathrm{nmol} \cdot \mathrm{kg}^{-1}$ per $4 \mathrm{~h}$ ).

\section{Discussion}

Both glucose and insulin regulate hepatic and muscle glucose metabolism (11-16). Whereas numerous studies have demonstrated that people with NIDDM are insulin resistant, the contribution of insulin resistance to carbohydrate intolerance under physiological conditions remains uncertain. Furthermore, because the ability of glucose per se to inhibit glucose production and to stimulate glucose utilization is dependent upon the prevailing insulin concentrations (11-16), the extent to which the response to increasing glucose concentrations either compensates for or exacerbates insulin resistance in people with NIDDM is unknown. The present studies indicate that small amounts of insulin $(\sim 2 \mathrm{U})$, when given so as to mimic normal postprandial systemic insulin concentrations, have surprisingly potent effects on glucose metabolism. They result in prompt suppression of hepatic glucose release in both diabetic and nondiabetic subjects. In contrast, whereas insulin administered in this manner produces a brisk increase in glucose disposal in nondiabetic individuals, it has virtually no effect on glucose disposal in people with NIDDM. However, an increase in glucose concentration similar to that observed in nondiabetic subjects after ingestion of $50 \mathrm{~g}$ of carbohydrate markedly and comparably enhances glucose disposal in both groups. Although hyperglycemia only minimally further suppresses hepatic glu- 
cose release, the magnitude of this effect also was the same in the diabetic and nondiabetic subjects. These data indicate that although people with NIDDM are resistant to the effects of insulin, they are not resistant to the regulatory effects of glucose. These data also indicate that in the presence of physiological changes in insulin concentrations, there is a marked difference in the severity of hepatic and extrahepatic insulin resistance in NIDDM.

The effects of insulin on glucose metabolism in NIDDM. A variety of techniques have been used to assess insulin action in people with NIDDM (26). The hyperinsulinemic euglycemic clamp is perhaps the most widely used. When submaximal insulin concentrations are used, this method has shown repeatedly that people with NIDDM have both hepatic and extrahepatic insulin resistance (3-7). Although this method permits the response to a given insulin concentration to be quantitated, it necessitates maintenance of insulin concentrations constant for several hours, a circumstance rarely if ever observed under physiological conditions. We and others have shown that the response to insulin is greater when insulin is administered so as to produce changing rather than constant insulin concentrations (8-10). An enhanced response to changing insulin concentrations may account at least in part for the near normal pattern of suppression of hepatic glucose release observed in the diabetic subjects. It is possible that the overnight insulin infusion improved hepatic insulin action in the diabetic subjects. However, it did not normalize insulin action because the decrement in hepatic glucose release in response to the same increment in insulin was slightly (but significantly) less in the diabetic than nondiabetic subjects. In addition, we have demonstrated in a separate series of experiments that hepatic glucose release during a traditional hyperinsulinemic euglycemic clamp remains excessive after an overnight insulin infusion (26a). Finally, even if the nocturnal infusion improved hepatic insulin action, it clearly did not restore the extrahepatic response to insulin.

The marked disparity between the degree of hepatic and extrahepatic insulin resistance is intriguing. Recent studies have demonstrated that insulin must traverse an endothelial barrier to reach the interstitial fluid that bathes muscle (27). In contrast, insulin presumably has ready access to the liver via the large fenestrations present in the portal venous system (28). However, the difference in the hepatic and extrahepatic response to insulin in the diabetic subjects is unlikely to be solely due to slowed equilibration between blood and interstitial insulin concentrations because this presumably would be associated with a delayed rather than a virtually absent response to insulin. Alternatively the discordance between the severity of hepatic and extrahepatic insulin resistance may be due to differences in liver and muscle insulin binding and/or signal transduction (29-31). Insulin increases glucose uptake in muscle primarily by increasing the translocation of glucose transporters to the plasma membrane whereas it decreases hepatic glucose release by altering the activity of glycogenolytic and gluconeogenic enzymes $(13,32)$. Therefore, a defect in a signaling pathway (e.g., glucose transport) present in muscle but not in the liver could readily explain the present results.

Regardless of the mechanism(s), the near normal suppression of hepatic glucose release by insulin in the diabetic subjects in the face of virtually no change in glucose disposal is of considerable interest in view of recent data from nondiabetic animals that suggest a causal relationship between these two processes $(33,34)$. If insulin's effects on liver and muscle glucose metabolism indeed are mediated through a single ratelimiting step in nondiabetic humans, this relationship clearly is altered in people with NIDDM.

The effects of glucose per se on glucose metabolism in NIDDM. In previous studies in a separate group of subjects, we have demonstrated that the same glucose and insulin profiles as used in the hyperglycemic portion of the present experiments resulted in normal suppression of hepatic glucose release and substantial, albeit not fully normal, stimulation of glucose uptake in people with NIDDM (19). The present experiments confirm these observations. However, because both glucose and insulin increased concurrently in our previous experiments, we could not distinguish abnormalities due to alterations in insulin action from those due to alterations in the effectiveness of glucose. This question was addressed in the present experiments by studying the same individuals twice under identical conditions with the exception that on one occasion euglycemia was maintained whereas on the other occasion a postprandial glucose profile was produced. Whereas the insulin infusion had essentially no effect on glucose disposal in the diabetic subjects when euglycemia was maintained, in the presence of hyperglycemia it resulted in an increase in glucose disposal to rates similar to those observed in the nondiabetic subjects during insulin alone (Fig. 4). In contrast, insulin had a marked inhibitory effect on hepatic glucose release in both groups with suppression being only modestly enhanced by hyperglycemia. Of perhaps greatest interest, despite the marked differences in insulin action, the effectiveness of glucose, calculated by subtracting the results observed during the euglycemic experiments from those observed during the hyperglycemic experiments, did not differ in the diabetic and nondiabetic subjects (Fig. 7).

Previous investigations of glucose effectiveness in NIDDM have yielded results that at first glance appear to be contradictory (35-37). Capaldo et al. (35) reported that glucose effectiveness in the presence of insulin deficiency is normal whereas Baron et al. (36) reported that it is increased. On the other hand, Welch et al. (37) reported that glucose effectiveness in the presence of basal insulin concentrations is decreased. These apparent inconsistencies likely are due to the fact each investigator used a different strategy to try to factor out the effects of glucose from those of insulin.

It should be noted that the definition and measurement of glucose effectiveness used in the present study is different from, albeit related to, that used by these investigators $(26,35-37)$. First, we have examined both hepatic and peripheral glucose effectiveness. Hepatic glucose effectiveness is defined in this study as the difference between hepatic glucose release observed during the hyperglycemic and euglycemic experiments. Peripheral glucose effectiveness is defined as the difference between glucose disposal or forearm $\mathrm{A}-\mathrm{V}$ glucose extraction during the hyperglycemic and euglycemic experiments. Second, and perhaps more importantly, glucose effectiveness in the present experiments was measured under nonsteady state conditions during a prandial insulin profile. Because insulin is continuously changing in the present study, each index of glucose effectiveness (hepatic/extrahepatic) does not refer to a single fixed level of insulin $(26,35-37)$. This distinction is important because in the presence of basal insulin concentrations non-insulindependent tissues (e.g., gut, brain) account for the majority of glucose disposal. In contrast, during a prandial rise in insulin, the majority of glucose is taken up by insulin-sensitive tissues 
(e.g., muscle). In addition, previous investigations have shown that the effect of glucose on its own disposal in both nondiabetic humans and people with type 1 diabetes mellitus is dependent on insulin (14-16); an increment in glucose concentration results in a greater increment in glucose disposal when insulin concentrations are high than when insulin concentrations are low (14-16). Under normal living conditions, an increase in glucose concentrations in nondiabetic individuals is invariably accompanied by a concomitant increase in insulin. The present experimental design was therefore chosen in an effort to gain insight into the interaction between glucose and insulin under physiological conditions.

If insulin indeed modulates glucose effectiveness, how then can insulin facilitation of glucose effectiveness be normal in NIDDM when the ability of insulin to stimulate glucose uptake at any given glucose concentration is impaired? Glucose effectiveness in peripheral tissues represents the ability of glucose to enhance its own disappearance at a given level of insulin. Insulin sensitivity can be viewed as the ability of insulin to enhance glucose effectiveness at a given glucose concentration. In non-steady state, such as in the present experiments, glucose effectiveness represents the sum of at least two components: one is the glucose effectiveness at basal insulin concentrations and the other is the enhancement of glucose effectiveness due to insulin action. When both glucose and insulin concentrations are changing, the sum of these two components is time varying. Our measure of glucose effectiveness is related to the integral of this time-varying glucose effectiveness. The finding that diabetic subjects have normal glucose effectiveness despite marked insulin resistance indicates that the interaction between glucose and insulin differs in the diabetic and nondiabetic subjects. These data imply that hyperglycemia in some way compensates for the inability of insulin to stimulate glucose uptake. This postulated effect of hyperglycemia per se might occur either independently from insulin or synergistically with insulin. The hypothesis of a compensatory effect of hyperglycemia in NIDDM is in good agreement with the results previously reported by Revers et al. (38) derived during hyperinsulinemic euglycemic and hyperglycemic glucose clamps.

Glucose effectiveness could compensate for insulin resistance via several mechanisms. First, glucose effectiveness at basal insulin concentrations could be higher in diabetic than nondiabetic subjects. This supposition is not supported by the previous report by Welch et al. (37) that glucose effectiveness at basal insulin concentrations is decreased rather than increased. Second, hyperglycemia in itself could modulate glucose effectiveness by enhancing intracellular enzyme activity (e.g., glycogen synthase activity) $(39,40)$. However, an alteration in intracellular glucose metabolism presumably would not influence glucose uptake if glucose transport/phosphorylation remained rate limiting. Data have been presented arguing both for (41, 42) and against (43) transport/phosphorylation as the rate-limiting step for glucose uptake in NIDDM during insulin infusion. Third, although transport may remain rate limiting, hyperglycemia and hyperinsulinemia may facilitate glucose uptake via different transporters. For example, whereas insulin may act by increasing the number/activity of transporters with a low $K_{\mathrm{m}}$ (e.g., glut-4 transporters) for glucose (for review see reference 44), hyperglycemia may increase glucose uptake predominantly via transporters with a higher $K_{\mathrm{m}}$ (e.g., glut-1) for glucose (45). Fourth, hyperglycemia could increase the number or type of transporters (46). If so, acute increases in glucose and insulin must have different effects on these transporters. This postulate is consistent with the recent report by Dimitrakoudis et al. (47) that chronic hyperglycemia increases the number of glut- 1 but not glut-4 transporters in muscle. If indeed hyperglycemia is able to compensate for defects in insulin action, then multiple factors could contribute to "glucose effectiveness." Future studies will be required to determine the relative contribution of each of these mechanisms to the preservation of normal glucose effectiveness in NIDDM.

Limitations. It is impossible to prove that "glucose effectiveness" in the presence of prandial insulin concentrations is the same in diabetic and nondiabetic subjects. Using the current experimental design, we have found no evidence that the ability of glucose to facilitate its own uptake and to inhibit its own release is impaired in NIDDM. Although it is possible that methods used were not sufficiently sensitive to detect a true difference, we doubt that this is the case for several reasons. First, we could readily detect differences in insulin action between groups during both the euglycemic and hyperglycemic experiments. Second, the conclusions were concordant whether glucose effectiveness was assessed using the isotope dilution or the forearm catheterization techniques. Although the forearm data are reported as the $\mathrm{A}-\mathrm{V}$ difference, expression as forearm glucose uptake (i.e., multiplying the $\mathrm{A}-\mathrm{V}$ difference by "noisy" blood flows) yielded the identical conclusion. Third, there was extensive overlap between the diabetic and nondiabetic groups in the ability of glucose to enhance its own uptake; the ability of glucose to inhibit its own release, if anything, was greater in the diabetic than nondiabetic subjects. Therefore, although it is possible that we failed to detect a true impairment in glucose effectiveness in NIDDM, if so, the magnitude of such a defect is likely small.

Measurement of glucose turnover during non-steady state is problematic. Several recent studies have demonstrated that nonsteady state errors can be avoided by preventing rapid changes in glucose specific activity $(20,48,49)$. Although glucose specific activity increased slightly during the present experiments, these slight changes in specific activity introduce minimal or no error in measurement of turnover $(20,48,49)$. In addition, the pattern of change in specific activity was the same in the diabetic and nondiabetic subjects.

An overnight insulin infusion was used in the diabetic subjects to ensure that the glucose concentrations were the same in both groups before and during the prandial insulin infusions. The effect, if any, of this overnight insulin infusion on insulin action currently is not known. Glucagon concentrations were maintained constant and equal in both groups during all experiments by infusing somatostatin and replacement amounts of glucagon. This differs from the normal situation in which fasting glucagon concentrations tend to be higher and in which suppression after carbohydrate ingestion tends to be lower in diabetic than nondiabetic subjects $(50,51)$. Although the present experiments indicate that the hepatic response to a "physiological" insulin profile is only minimally impaired, inappropriately elevated glucagon concentrations that are observed in diabetic people after food ingestion may magnify this defect.

In contrast to what occurs after food ingestion, glucose and insulin were infused into the peripheral rather than the portal venous systems. Somatostatin infusion resulted in essentially complete inhibition of insulin secretion in both groups of subjects. Although the prandial insulin infusions were identical in both groups, the absolute insulin levels were higher in the dia- 
betic subjects because of their higher basal insulin concentrations $(19,52)$. On the other hand, the increment in systemic and therefore presumably portal venous insulin concentrations produced by the prandial insulin infusions was the same in the diabetic and nondiabetic subjects. Although not directly measured, we doubt that hepatic glucose uptake is impaired in NIDDM because previous experiments examining splanchnic glucose extraction after ingestion of radiolabeled meals found no evidence for such a defect in either diabetic animals or humans $(21,53,54)$.

Summary and conclusions. The present experiment clearly demonstrate a discordance between the severity of hepatic and extrahepatic insulin resistance in people with NIDDM. Whereas administration of insulin in a manner that produced continuously changing insulin concentrations had no effect on glucose disposal, it resulted in prompt suppression of hepatic glucose release. In contrast, glucose disposal was markedly stimulated when the increase in insulin was accompanied by an increase in glucose concentration. Despite the presence of insulin resistance in the diabetic subjects, the ability of glucose to facilitate its own uptake and to suppress it own release was not impaired. Thus, although restoration of insulin secretion in people with NIDDM may be accompanied by a normal hepatic response, extrahepatic carbohydrate metabolism is likely to remain abnormal. These data also suggest that new therapies should be directed primarily toward improvement of extrahepatic insulin action rather than altering hepatic responsiveness to insulin or glucose effectiveness. The mechanism that permits normal glucose but abnormal insulin facilitation of glucose uptake in people with NIDDM requires further investigation.

\section{Acknowledgments}

We thank E. Butkowicz, J. King, T. Madson, D. Nash, and Dr. M. Joyner for their technical assistance; A. Pelot for assistance in typing the manuscript; and the staff of the Clinical Research Center for assistance in performing the studies.

This work was supported by U. S. Public Health Service (DK29953, RR00585), the Mayo Foundation, and Ministero Universita Ricerca Scientifica Tecnica (Murst) (Italy). Dr. Aus A. Alzaid was supported by a mentor-based American Diabetes Association Research Fellowship Award and by the Armed Forces Hospital, Riyadh, Saudi Arabia.

\section{References}

1. Reaven, G., R. Bernstein, B. Davis, and J. Olefsky. 1976. Nonketotic diabetes mellitus: insulin deficiency or insulin resistance? Am. J. Med. 60:80-88.

2. Bugardus, C., S. Lillioja, B. Howard, G. Reaven, and D. Mott. 1984. Relationships between insulin secretion, insulin action, and fasting plasma glucose concentration in nondiabetic and noninsulin-dependent diabetic subjects. J. Clin. Invest. 74:1238-1246.

3. Kolterman, O., R. Gray, J. Griffin, P. Burstein, J. Insel, J. Scarlett, and J. Olefsky. 1981. Receptor and post receptor defects contribute to the insulin resistance in non-insulin dependent diabetes mellitus. J. Clin. Invest. 68:957-965.

4. Groop, L. C., R. C. Bonadonna, S. DelPrato, K. Ratheiser, K. Zyck, E. Ferrannini, and R. A. DeFronzo. 1989. Glucose and free fatty acid metabolism in non-insulin-dependent diabetes mellitus. Evidence for multiple sites of insulin resistance. J. Clin. Invest. 84:205-213.

5. Firth, R., P. Bell, and R. Rizza. 1986. Effects of tolazamide and exogenous insulin on insulin action in patients with non-insulin-dependent diabetes mellitus. N. Engl. J. Med. 314:1280-1286.

6. Campbell, P., L. Mandarino, and J. Gerich. 1988. Quantification of the relative impairment of actions of insulin on hepatic glucose production and peripheral glucose uptake in non-insulin-dependent diabetes mellitus. Metab. Clin. Exp. 37:15-21.

7. Butler, P., A. Caumo, A. Zerman, P. O'Brien, C. Cobelli, and R. Rizza. 1993. Methods for assessment of the rate of onset and offset of insulin action during nonsteady state in humans. Am. J. Physiol. 264:E548-E560.
8. Doeden, B., and R. Rizza. 1987. Use of a variable insulin infusion to assess insulin action in obesity: defects in both the kinetics and amplitude of response. J. Clin. Endocrinol. Metab. 64:902-908.

9. Matthews, D., B. Naylor, R. Jones, G. Ward, and R. Turner. 1983. Pulsatile insulin has greater hypoglycemic effect than continuous delivery. Diabetes. 32:617-621.

10. Paolisso, G., A. Scheen, D. Giugliano, S. Sgambato, A. Albert, M. Varricchio, F. D’Onofrio, and P. Lefebvre. 1991. Pulsatile insulin delivery has greater metabolic effects than continuous hormone administration in man: importance of pulse frequency. J. Clin. Endocrinol. Metab. 72:607-615.

11. Rizza, R., P. Cryer, M. Haymond, and J. Gerich. 1980. Adrenergic mechanisms for the effects of epinephrine on glucose production and clearance in man. J. Clin. Invest. 65:682-689.

12. Sacca, L., P. Cryer, and R. Sherwin. 1979. Blood glucose regulates the effects of insulin and counterregulatory hormones on glucose production in vivo. Diabetes. 28:533-536.

13. Youn, J., M. Youn, and R. Bergman. 1986. Synergism of glucose and fructose in net glycogen synthesis in perfused rat livers. J. Biol. Chem. 261:15960-15969.

14. Hansen, I., P. Cryer, and R. Rizza. 1985. Comparison of insulin-mediated and glucose-mediated glucose disposal in patients with insulin-dependent diabetes mellitus and in non-diabetic subjects. Diabetes. 34:751-755.

15. Best, J., G. Taborsky, J. Halter, and D. Porte. 1981. Glucose disposal is not proportional to plasma glucose level in man. Diabetes. 30:847-850.

16. Yki-Jarvinen, H., D. Mott, A. Young, K. Stone, and C. Bogardus. 1987. Regulation of glycogen synthase and phosphorylase activities by glucose and insulin in human skeletal muscle. J. Clin. Invest. 80:95-100.

17. Shuster, L., V. Go, R. Rizza, P. O'Brien, and J. Service. 1988. Incretin effect due to increased secretion and decreased clearance of insulin in normal humans. Diabetes. 37:200-203.

18. White, N., D. Skor, and J. Santiago. 1982. Practical closed-loop insulin delivery. A system for the maintenance of overnight euglycemia and the calculation of basal insulin requirements insulin-dependent diabetes. Ann. Intern. Med. 97:210-213.

19. Katz, H., M. Homan, M. Jensen, A. Caumo, C. Cobelli, and R. Rizza. 1994. Assessment of insulin action in NIDDM in the presence of dynamic changes in insulin and glucose concentration. Diabetes. 34:289-296.

20. Finegood, D., R. Bergman, and M. Vranic. 1987. Estimation of endogenous glucose production during hyperinsulinemic-euglycemic glucose clamps: comparison of unlabelled and labelled exogenous glucose infusates. Diabetes. 36:914924.

21. Firth, R., P. Bell, H. Marsh, I. Hansen, and R. Rizza. 1986. Postprandial hyperglycemia in patients with non-insulin-dependent diabetes mellitus. J. Clin. Invest. 77:1525-1532.

22. Butler, P., E. Kryshak, and R. Rizza. 1991. Mechanism of growth hormoneinduced postprandial carbohydrate intolerance in humans. Am. J. Physiol. 260:E513-E520.

23. Bradley, D. C., G. M. Steil, and R. N. Bergman. 1993. Quantification of measurement error with optimal segments: basis for adaptive time course smoothing. Am. J. Physiol. 264:E902-E911.

24. Radziuk, J., K. Norwich, and M. Vranic. 1978. Experimental validation of measurements of glucose turnover in nonsteady state. Am. J. Physiol. 234:E84E93.

25. Carson, E., C. Cobelli, and L. Finkelstein. 1983. The Mathematical Modeling of the Endocrine-Metabolism System. Wiley, New York. 189-193.

26. Bergman, R., D. Finegood, and M. Ader. 1985. Assessment of insulin sensitivity in vivo. Endocr. Rev. 6:45-86.

26a. Turk, D., A. Alzaid, S. Dinneen, K. S. Nair, and R. Rizza. 1995. The effects of non-insulin dependent diabetes mellitus (NIDDM) on the kinetics of onset of insulin action in hepatic and extrahepatic tissues. J. Clin. Invest. In press.

27. Yang, Y., I. Hope, M. Ader, and R. Bergman. 1989. Insulin transport across capillaries is rate limiting for insulin action in dogs. J. Clin. Invest. 84:1620-1628.

28. Bliar, H. 1949. A re-examination of the structure of mammalian liver. Am. J. Anat. 85:379-456.

29. Burant, C., M. Treutelaar, N. Block, and M. G. Buse. 1986. Structural differences between liver- and muscle-derived insulin receptors in rats. J. Biol. Chem. 261:14361-14364.

30. Caro, J., S. Raju, M. Sinha, I. Goldfine, and G. Dohm. 1988. Heterogeneity of human liver, muscle, and adipose tissue insulin receptor. Biochem. Biophys. Res. Commun. 151:123-129.

31. Kellerer, M., G. Sesti, E. Seffer, B. Obermaier-Kusser, D. Pongratz, L. Mosthaf, and H. Häring. 1993. Altered pattern of insulin receptor isotypes in skeletal muscle membranes of type 2 (non-insulin-dependent) diabetic subjects. Diabetologia. 36:628-632.

32. Rosetti, L., A. Giaccari, N. Barzilai, K. Howard, G. Sebel, and M. Hu. 1993. Mechanisms by which hyperglycemia inhibits hepatic glucose production in conscious rats. Implications for the pathogenesis of fasting hyperglycemia in diabetes. J. Clin. Invest. 92:1126-1134.

33. Bradley, D. C., R. A. Poulin, and R. N. Bergman. 1993. Dynamics of 
hepatic and peripheral insulin effects suggest common rate-limiting step in vivo. Diabetes. 42:296-306.

34. Giacca, A., J. Fisher, Z. Shi, R. Gupta, H. Lickley, and M. Vranic. 1992. Importance of peripheral insulin levels for insulin-induced suppression of glucose production in depancreatized dogs. J. Clin. Invest. 92:1769-1777.

35. Capaldo, B., D. Santoro, G. Riccardi, N. Perotti, and L. Sacca. 1986. Direct evidence for a stimulatory effect of hyperglycemia per se on peripheral glucose disposal in type II diabetes. J. Clin. Invest. 77:1285-1290.

36. Baron, A., G. Kolterman, J. Bell, L. Mandarino, and J. Olefsky. 1985 Rates of noninsulin-mediated glucose uptake are elevated in type II diabetic subjects. J. Clin. Invest. 76:1782-1788.

37. Welch, S., S. Gebhan, S., R. Bergman, and L. Phillips. 1990. Minimal model analysis of intravenous glucose tolerance test-derived insulin sensitivity in diabetic subjects. J. Clin. Endocrinol. Metab. 71:1508-1549.

38. Revers, R. R., R. Fink, J. Griffin, J. M. Olefsky, and O. G. Kolterman. 1984. Influence of hyperglycemia on insulin's in vivo effects in type II diabetes. J. Clin. Invest. 73:664-672.

39. Mandarino, L. J., A. Consoli, D. E. Kelley, J. J. Reilly, and N. Nurjhan 1990. Fasting hyperglycemia normalizes oxidative and nonoxidative pathways of insulin-stimulated glucose metabolism in noninsulin-dependent diabetes mellitus. J. Clin. Endocrinol. Metab. 71:1544-1551.

40. Lavoie, L., D. Dimitrakoudis, A. Marette, B. Annabi, A. Klip, M. Vranic, and G. Van de Werve. 1993. Opposite effects of hyperglycemia and insulin deficiency on liver glycogen synthase phosphatase activity in the diabetic rat. Diabetes. 42:363-366.

41. Rothman, D., R. Shulman, and G. Shulman. 1992. ${ }^{31} \mathrm{P}$ Nuclear magnetic resonance measurements of muscle glucose-6-phosphate. Evidence for reduced insulin-dependent muscle glucose transport or phosphorylation activity in noninsulin-dependent diabetes mellitus. J. Clin. Invest. 89:1069-1075.

42. Butler, P. C., E. J. Kryshak, M. Marsh, and R. A. Rizza. 1990. Effect of insulin on oxidation of intracellularly and extracellularly derived glucose in patients with NIDDM. Evidence for primary defect in glucose transport and/or phosphorylation but not oxidation. Diabetes. 39:1373-1380.

43. Vaag, A., J. Henriksen, and H. Beck-Neilsen. 1992. Decreased insulin activation of glycogen synthase in skeletal muscles in young nonobese caucasian first-degree relatives of patients with non-insulin-dependent diabetes mellitus. $J$. Clin. Invest. 89:782-788.

44. Kahn, B. 1992. Facilitative glucose transporters. Regulatory mechanisms and dysregulation in diabetes. J. Clin. Invest. 89:1367-1374.

45. Nishimura, H., F. V. Pallardo, G. A. Seidner, S. Vannucci, I. A. Simpson, and M. J. Birnbaum, 1993. Kinetics of GLUT1 and GLUT4 glucose transporters expressed in Xenopus oocytes. J. Biol. Chem. 268:8514-8520.

46. Harik, S. I., R. A. Behmand, and B. M. Arafah. 1991. Chronic hyperglycemia increases the density of glucose transporters in human erythrocyte membranes. J. Clin. Endocrinol. Metab. 72:814-818.

47. Dimitrakoudis, D., M. Vranic, and A. Klip. 1992. Effects of hyperglycemia on glucose transporters of the muscle: Use of the renal glucose reabsorption inhibitor phlorizin to control glycemia. J. Am. Soc. Nephrol. 3:1078-1091.

48. Cobelli, C., A. Mari, and E. Ferrannini. 1987. Non-steady state: error analysis of Steele's model and developments for glucose kinetics. Am. J. Physiol. 252:E679-E689.

49. Butler, P., A. Caumo, A. Zerman, P. O'Brien, C. Cobelli, and R. Rizza. 1993. Methods for assessment of the rate of onset and offset of insulin action during nonsteady state in man. Am. J. Physiol. 264:E548-E560.

50. Yamamoto, T., P. Raskin, I. Aydin, and R. Unger. 1979. Effects of insulin on the response of immunoreactive glucagon to an intravenous glucose load in human diabetes. Metab. Clin. Exp. 28:568-574.

51. Butler, P., and R. Rizza. 1991. Contribution to postprandial hyperglycemia and effect on initial splanchnic glucose clearance of hepatic glucose cycling in glucose-intolerant or NIDDM patients. Diabetes. 40:73-81.

52. Kelley, D., M. Mokan, and L. Mandarino. 1992. Intra-cellular defects in glucose metabolism in obese patients with NIDDM. Diabetes. 41:698-706.

53. Ferrannini, E., D. Simonson, L. Katz, G. Reichard, S. Bevilacqua, E. Barrett, M. Olsson, and R. DeFronzo. 1988. The disposal of an oral glucose load in patients with non-insulin-dependent diabetes. Metab. Clin. Exp. 37:79-85.

54. Chen, C., P. Williams, and I. Caterson. 1993. Liver and peripheral tissue glycogen metabolism in obese mice: effect of a mixed meal. Am. J. Physiol. 265:E743-E751 